Fax: + 442078863259

E-mail: panda@doctors.org.uk

Eye (2006) 20, 1446-1447. doi:10.1038/sj.eye.6702311; published online 3 March 2006

Sir,

\section{Valsalva retinopathy associated with idiopathic thrombocytopenic purpura and positive antiphospholipid antibodies}

Valsalva retinopathy may occur during a sudden increase in intraocular venous pressure caused by forced exhalation against a closed glottis.

Patients with idiopathic thrombocytopenic purpura (ITP) and positive antiphopholipid antibodies (aPL) are at particular risk for developing both bleeding and thrombotic complications. ${ }^{1}$

\section{Case report}

A 32-year-old female patient presented with sudden visual loss in the right eye during sexual intercourse. There was no past ocular history of note but she was known to have ITP with positive aPL. On presentation, her best corrected visual acuities (BCVA) were 6/36 in the right eye and $6 / 5$ in the left. The anterior segments were normal, but in the right eye there was a submacular trilaminar haemorrhage (subretinal, intraretinal, and subhyaloid) involving the superior arcade. (Figure 1a). Fundus biomicroscopy of the left eye was normal. Systemically, she was well and was on no medications. The blood pressure was $100 / 70 \mathrm{mmHg}$ on presentation. All blood tests were normal except for raised IgM anticardiolipin antibody consistent with her past medical history. Her platelet count was $195 \times 10^{3} / \mathrm{mm}^{3}$.

Within a few days, there was further reduction of vision with the BCVA dropping to 2/60. A fluorescein angiography (FA) and indocyanine green angiography (ICG) were performed. (Figure $1 \mathrm{~b}-\mathrm{d}$ ). Both examinations showed an area of hypofluorescence corresponding to the haemorrhage but no underlying neovascularization. The diagnosis of Valsalva retinopathy was made and all management options were explained. The patient elected not to undergo any surgical treatment. On subsequent follow-up at 3 weeks, 2, 4, and 6 months, the haemorrhage gradually absorbed leaving an area of atrophy of the retinal pigment epithelium (RPE) under the right fovea and an epiretinal membrane along the superior arcade (Figure $2 \mathrm{a}-\mathrm{d}$ ). Her BCVA remained at $6 / 60$.

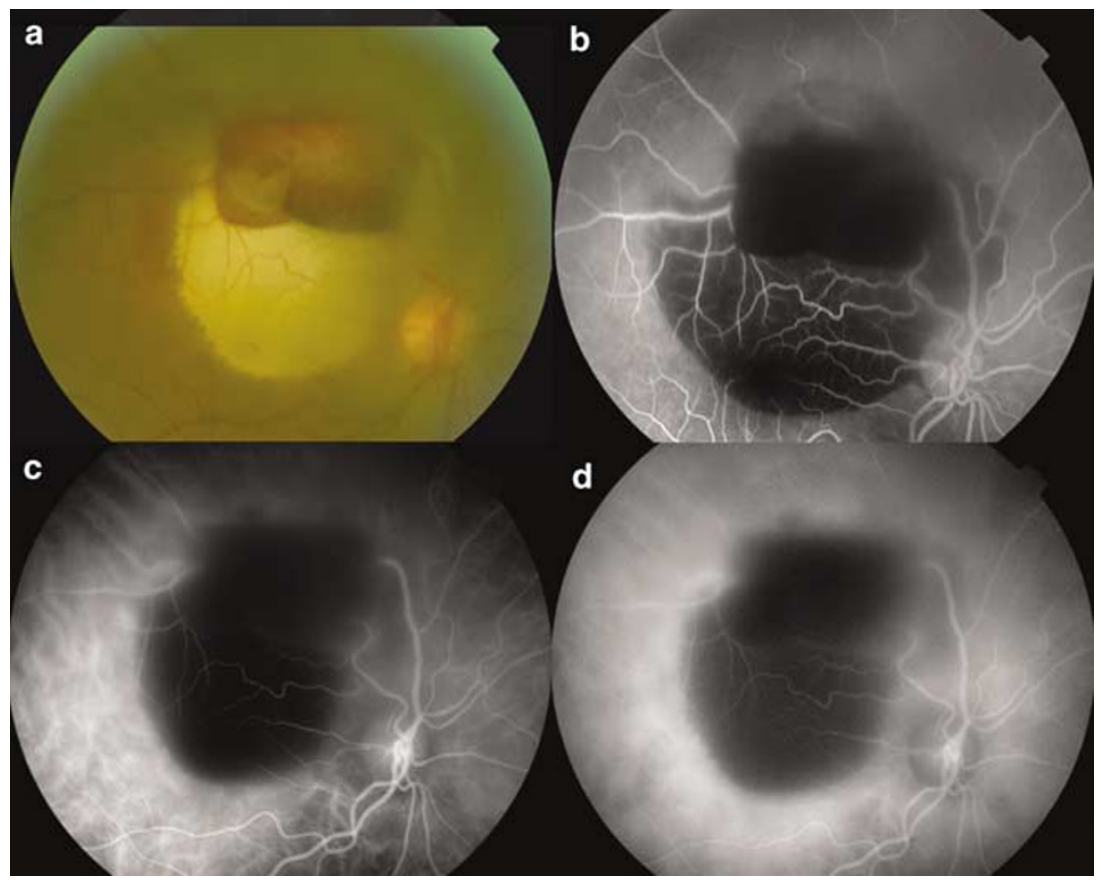

Figure 1 (a) Colour fundus picture on presentation showing the trilaminar submacular haemorrhage extending up to the superior arcade. (b) Fluorescein angiography showing an area of hypofluorescence corresponding to the haemorrhage. (c, d) Indocyanine green angiography showing an area of hypofluorescence corresponding to the haemorrhage but no underlying neovascularization. 


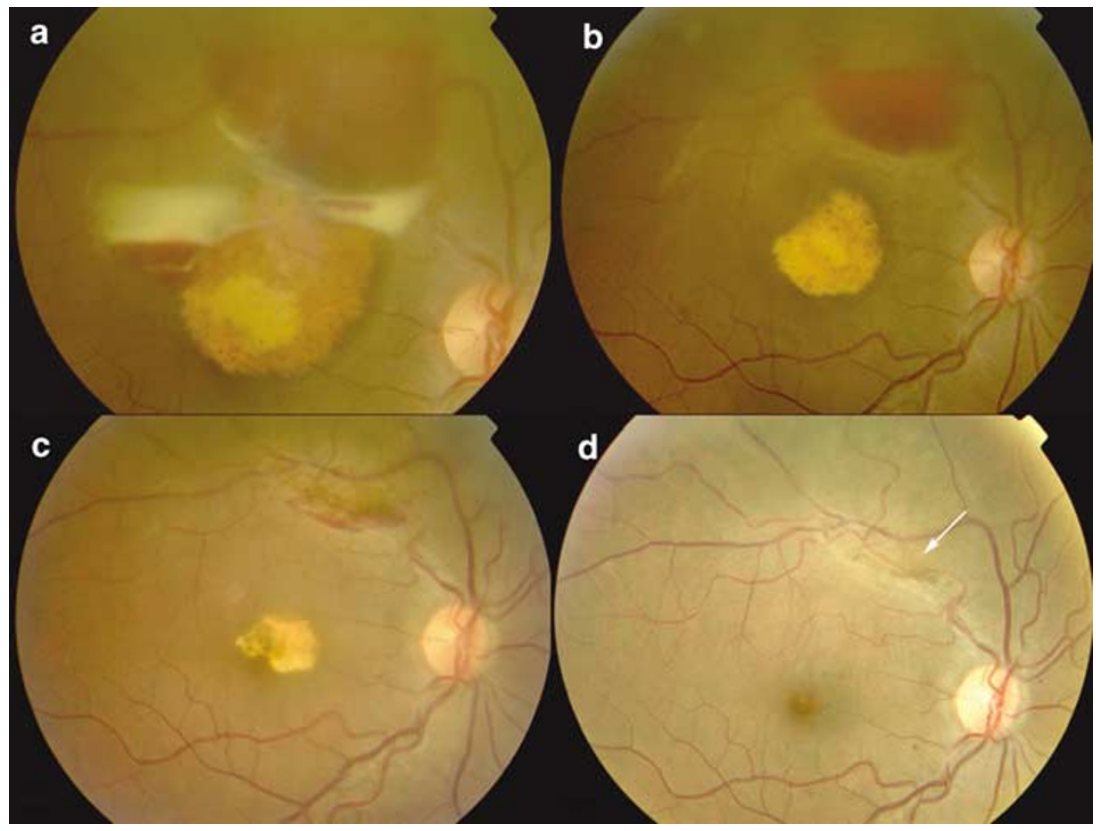

Figure 2 (a-c) Colour fundi pictures 3 weeks, 2, and 4 months later showing gradual absorption of the haemorrhage leaving an area of atrophy of the RPE under the fovea. (d) Colour fundus picture 6 months later showing complete absorption of the haemorrhage leaving an epiretinal membrane along the superior arcade (white arrow).

\section{Comment}

Valsalva retinopathy is characterized by the presence of a pre-retinal haemorrhage following a Valsalva maneuver and in most cases resolves spontaneously with favourable visual outcome.

A total of $30 \%$ of patients with ITP are positive for $\mathrm{aPL}^{2}$ and are at particular risk of developing both bleeding and thrombotic complications. ${ }^{1}$ Patients with primary antiphospholipid syndrome alone are more likely to present with ocular vaso-occlusive accidents involving the retinal and choroidal vessels. ${ }^{3}$ It is known that aPL are directed against cell membranes ${ }^{2}$ and they play a role in mediating platelet activation and aggregation. However, the exact role of aPL in the pathophysiology of thrombocytopenia remains obscure. ${ }^{1}$

Ocular associations with ITP include hemianopia secondary to intracranial haemorrhage and massive subretinal haemorrhage in association with age-related macular degeneration. ${ }^{4}$

The association between sudden loss of vision owing to retinal haemorrhage and sexual intercourse has been previously reported. ${ }^{5}$

The extent and trilaminar nature of the haemorrhage and the severity of retinal damage in this case were probably contributed by the presence of ITP with positive aPL.

In conclusion, this case highlights the severity of Valsalva retinopathy in association with ITP and positive aPL. We are not aware of any previous reports in spite of a medline search that was conducted by us.

\section{References}

1 Atsumi T, Furukawa S, Amengual O, Koike T. Antiphospholipid antibody associated thrombocytopenia and the paradoxical risk of thrombosis. Lupus 2005; 14: 499-504.

2 Hughes GRV. The antiphospholipid syndrome: ten years on. Lancet 1993; 342: 341-344.

3 Miserocchi E, Baltatzis S, Foster CS. Ocular features associated with anticardiolipin antibodies. A descriptive study. Am J Ophthalmol 2001; 131(4): 451-456.

4 Inoue T, Yanagi Y, Tamaki Y, Kami J, Kato Y. Massive subretinal haemorrhage secondary to age-related macular degeneration in a patient with idiopathic thrombocytopenic purpura. Eye 2004; 18(6): 656-657.

5 Friberg TR, Braunstein RA, Bressler NM. Sudden visual loss associated with sexual activity. Arch Ophthalmol 1995; 113: 738-742.

D Karagiannis and Z Gregor

Moorfields Eye Hospital, London, UK

Correspondence: D Karagiannis,

Moorfields Eye Hospital,

162 City Road,

London EC1V 2PD, UK

Tel: +44 778975 5930; 
Fax: + 4402075662972

E-mail: dimitrioskaragiannis@doctors.org.uk

Eye (2006) 20, 1447-1449. 10.1038/sj.eye.6702313; published online 10 March 2006

Sir,

\section{Routine follow-up after YAG laser capsulotomy for posterior capsule opacification}

A national survey found $63 \%$ of consultant ophthalmologists review their patients within the first 8 weeks after YAG laser capsulotomy for posterior capsule opacification (RCO Annual Congress Abstract, Goyal and Goel, 2003). Review after YAG laser capsulotomy is not a stipulation of current national guidelines. ${ }^{1}$ What evidence is there that follow-up is necessary?

A retrospective assessment of patient notes for 121 YAG laser capsulotomies at Queen Mary's Hospital Sidcup from July 2003 to March 2004 revealed that the vision in $81.7 \%$ of eyes was $6 / 9$ or better, where as $15.0 \%$ did not achieve $6 / 9$ due to a clear comorbidity. Of the remaining $3.3 \%$ with vision less than $6 / 9$, one patient had an inadequate capsule gap treated successfully with further laser, where as three patients had moderately reduced vision $(6 / 12-6 / 18)$ in the absence of a definitive diagnosis.

No complications were recorded at the time of laser or on review, including the $19 \%$ of cases at higher risk of complications (axial myopia, previous retinal detachment, lattice degeneration, atrophic retinal holes, glaucoma, sulcus fixated lens, or uveitis).

Despite the relatively small numbers assessed, the high rate of satisfactory outcomes and the absence of complications concur with findings already published, ${ }^{2,3}$ which affirm the efficacy and safety of YAG laser capsulotomy. Given an adequate capsulotomy gap, minimising of lens pitting, and an accurate refraction, visual outcomes for laser should be excellent, in the absence of a comorbid condition.

Even the more common complications, namely retinal detachment and cystoid macular oedema, which have an incidence of approximately $1: 100$, tend to occur after the first few months, ${ }^{4,5}$ by which time most patients without a concurrent ophthalmic problem will have been discharged from outpatient follow-up.

More infrequent complications (less than 1:600) include macular hole, cracked lens, vitreous prolapse into the anterior chamber, lens subluxation, hyphema, uveitis, pupil block, and exacerbation of local endophthalmitis, many of which, should they occur, are identifiable at the time of the laser treatment. $2,6,7$

The spike in intraocular pressure that may occur following YAG laser, of particular clinical importance in patients with glaucoma, happens in the hours immediately post-procedure. Therefore, patients should have their pressures checked and receive treatment, if required, during the same visit, rather than at a later clinic review.

In view of the available evidence, we feel routine review in the first few weeks after laser is not justifiable, and that clinical resources should be redirected to areas of greater clinical need. We affirm the national guidelines, which emphasise the importance of both warning patients about possible complications and providing an advice sheet. ${ }^{1}$ In the event of pain, an absence of improvement or indeed a worsening of vision, or symptoms of retinal detachment, patients should be encouraged to report urgently, whether it be days, weeks, months, or even years after laser treatment.

\section{References}

1 Royal College of Ophthalmologists. Management of posterior capsule opacification. In: Seward H (ed). Royal College of Ophthalmologists' Management of Cataract Surgery Guidelines London, 2004 p 34.

2 Flohr MJ, Robin AL, Kelley JS. Early complications following Q-switched neodymium: YAG laser posterior capsulotomy. Ophthalmology 1985; 92: 360-363.

3 Buehl W, Sacu S, Findl O. Association between intensity of posterior capsule opacification and visual acuity. J Cataract Refract Surg 2005; 31: 543-547.

4 Lee MS, Lass JH. Rapid response of cystoid macular edema related to Nd:YAG laser capsulotomy to $0.5 \%$ Ketorolac. Ophthalmic Surg Lasers Imaging 2004; 35: 162-164.

5 Steinert RF, Puliafito CA, Kumar SR, Dudak SD, Patel S. Cystoid macular edema, retinal detachment, and glaucoma after Nd:YAG laser posterior capsulotomy. Am J Ophthalmol 1991; 112: 373-380.

6 Winslow RL, Taylor BC. Retinal complications following YAG laser capsulotomy. Ophthalmology 1985; 92: 785-789.

7 Shah GR, Gills JP, Durham DG, Ausmus WH. Three thousand YAG lasers in posterior capsulotomies: an analysis of complications and comparison to polishing and surgical discission. Ophthalmic Surg 1986; 17: 473-477.

HJ Bunting ${ }^{1}$ and $\mathrm{H}^{2}$ Laganowski

'Department of Ophthalmology, University Hospital of Wales, Heath Park, Cardiff, Wales, UK

${ }^{2}$ Queen Mary's Hospital, Frognal Avenue Sidcup, UK

Correspondence: HJ Bunting, Cardiff Eye Unit, 